\title{
Performance Analysis of Orthogonal Code Hopping Multiplexing Systems
}

\author{
Bang Chul Jung, Hu Jin, Dan Keun Sung, and Sae-Young Chung \\ CNR Lab., Dept. of EECS., KAIST, 373-1, \\ Guseong-dong, Yuseong-gu, Daejeon, 305-701, KOREA \\ Email: bcjung@cnr.kaist.ac.kr; jinhu@cnr.kaist.ac.kr; dksung@ee.kaist.ac.kr; sychung@ee.kaist.ac.kr
}

\begin{abstract}
In orthogonal code hopping multiplexing (OCHM) systems, hopping pattern (HP) collisions may degrade the system performance. Previous studies on the effect of HP collisions in OCHM systems were mainly based on computer simulations and there was no rigorous mathematical analysis of bit error rate (BER) performance. The HP collisions in OCHM systems differ from the hits in frequency-hopping (FH) systems or intracell interference in DS-CDMA systems because it can be effectively controlled through synergy and perforation techniques. In this paper, we introduce a received signal model for OCHM systems and analyze the BER performance for OCHM systems. Through the analysis of the BER performance, OCHM systems can be characterized more clearly and the allocated power at base station can be estimated. Furthermore, the user capacity is analyzed for a given channel coding scheme.
\end{abstract}

\section{INTRODUCTION}

Recently, data traffic has gradually increased in wireless communication systems. From this trend, data traffic will be expected to be dominant in future wireless systems. Data traffic is inherently bursty and generally exhibits low user activity. Furthermore, there is more downlink traffic than uplink traffic. Several efficient downlink systems have been proposed to provide this data traffic in wireless link [1], [2].

An orthogonal code hopping multiplexing (OCHM) system [3]-[7] has been proposed to accommodate a lager number of mobile users (MUs) with bursty traffic than the number of orthogonal codewords in downlink. It utilizes statistical multiplexing for orthogonal downlink in DS/CDMA systems. Since each MU communicates with base station (BS) through a given orthogonal code hopping pattern (HP), signaling messages for allocation and de-allocation of orthogonal codewords are not needed during a session. HP can be randomly generated based on an MU specific number, such as electronic serial number (ESN) and HP collisions between MUs may occur.

When an HP collision among MUs occurs in the conventional FH-CDMA systems, it is considered as an inevitable interference (hit) in case that all MUs are asynchronous with one another [8]. However, the HP collision can be detected and controlled by BS in a synchronous downlink environment. Furthermore, HP collisions in OCHM systems differ from the ICI in the uplink of DS-CDMA systems which is characterized by the cross-correlation function between non-orthogonal codewords.

Previous studies [3]-[7] on the OCHM systems have focused on the mitigation of the perforation effect. However, the performance of these proposed schemes was evaluated mainly based on computer simulations, especially, in terms of the frame error rate (FER) for given perforation probabilities and received $E_{b} / N_{0}$ values. There has been no rigorous mathematical analysis for the perforation effect on the system performance. In this paper, we introduce a received signal model in OCHM systems and analyze the BER performance. Through the analysis of BER, the FER performance at MU can be estimated and the user capacity of OCHM system for a given channel coding scheme is also analyzed. Furthermore, the allocated power for each MU at BS can be estimated.

The rest of this paper is organized as follows: In Section II, OCHM systems are briefly reviewed. The performance of OCHM systems is analyzed in Section III. Numerical examples for the performance of OCHM system are described in Section IV. Finally, conclusions are presented in Section V.

\section{BACKGROUND OF OCHM SYSTEMS}

\section{A. OCHM Mechanism, HP collisions, Perforation, and Syn-} ergy

The OCHM systems utilize a synergy and perforation scheme for HP collision control at the BS. Fig. 1 shows the block diagram of an OCHM system deploying the synergy and perforation scheme. Furthermore, both the transmitted and received power levels for a specific user are shown. $T_{s}$ denotes the symbol time. Each user changes the orthogonal codeword (OC) according to HP at each symbol time, during which a HP collision may occur. However, most of users may be inactive because of low channel activities. Users $b$ and $d$ are inactive in Fig. 1, but they follow their HPs during their sessions. In this case, HP collisions between an active user group and an inactive user group do not affect the performance of the active user group. The shaded parts in Fig. 1 indicate this type of collision.

When an HP collision among the active users occurs, BS compares the user data experiencing the HP collision and determines whether all user data with the same HP collision are the same or not. If all the corresponding data are the same, all the corresponding data symbols are transmitted (synergy) without control, which results in an energy gain at the receiver. On the contrary, if all data with the same HP collisions are not the same, all the corresponding data symbols are not transmitted (perforated) during the symbol time. For example, 


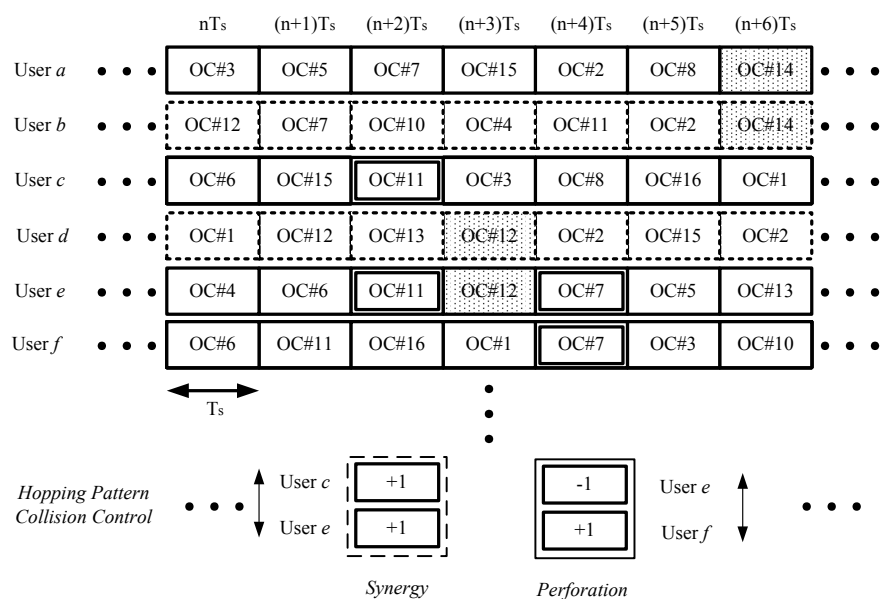

User $e$ 's power allocation at the BS

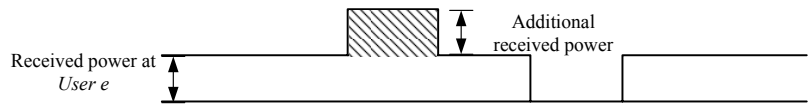

Fig. 1. Block diagram of the OCHM system

User $e$ experiences a synergy at $(n+2) T_{s}$ and a perforation at $(n+4) T_{s}$.

When a user experiences a synergy, BS allocates power for the user without any change, and then, all the symbols experiencing the synergy have an additional energy at the receivers because of other users' energy added by despreading process using the same OC. The quantity of the additional power is determined by the distance between a BS and codecollision users in case that the OCHM system utilizes a power control scheme. In Fig. 1, if User $e$ is at a cell boundary and User $c$ is located near a BS, the additional received power at User $e$ is much smaller than the average received power required which is the power when an HP collision does not occur. On the contrary, the additional received power at User $c$ is much bigger than the average received power in case of an HP collision. The synergy scheme results in an energy gain. However, its effect varies according to the location of users in a cell. Statistically, the users near BS have a more energy gain than those at the cell boundary. Therefore, in OCHM systems, the energy gain at the receiver due to the synergy scheme is complex to analyze even though several previous papers assumed all the HP-collision users are located in the same distance from BS in their performance evaluations [3][7].

When a user experiences a perforation, BS does not allocate power for the perforated symbol and the user detects only noise during the perforated symbol time. Hence, the perforation degrades the BER performance and some additional energy may be required to trasmit for a target BER.

The HP collision probability of the OCHM system is expressed as:

$$
P_{c}=1-\left(1-\frac{\bar{v}}{N_{O C}}\right)^{M-1}
$$

where $\bar{v}$ is the channel activity, $N_{O C}$ is the number of orthogonal codewords, and $M$ is the number of users in a cell. For a given channel activity $\bar{v}, P_{c}$ increases as the number of active users increases. The perforation probability of encoded symbols in the conventional OCHM systems is written as:

$$
P_{p}=1-\left(1-\frac{s-1}{s} \cdot \frac{\bar{v}}{N_{O C}}\right)^{M-1},
$$

where $s$ is the number of symbol locations in data modulation (i.e. $s=2$ for BPSK). Hence, the synergy probability is given as:

$$
P_{s}=P_{c}-P_{p}
$$

\section{B. Perforation Only Model (POM)}

In this paper, we assume the additional received power is set to zero. We call this signal model a perforation only model (POM) since it considers only a perforation scheme. Hence, POM provides an upper bound of the BER performance of OCHM systems. The received signal model of the BPSK symbol in AWGN channel is expressed as:

$$
Y= \begin{cases}t_{1} \sim N\left(0, \sigma^{2}\right), & \text { if perforation } \\ t_{2} \sim N\left(\sqrt{E_{s}}, \sigma^{2}\right), & \text { otherwise. }\end{cases}
$$

In Eq. (4), we also assume that a positive symbol is transmitted and its symbol energy is set to $E_{s}$. The distribution function of POM is obtained as

$$
F_{Y}(y)=G\left(\frac{y}{\sigma}\right) \cdot P_{p}+G\left(\frac{y-\sqrt{E_{s}}}{\sigma}\right) \cdot\left(1-P_{p}\right),
$$

where

$$
G\left(\frac{x-\mu}{\sigma}\right)=\int_{-\infty}^{x} \frac{1}{\sqrt{2 \pi \sigma^{2}}} e^{-(x-\mu)^{2} / 2 \sigma^{2}} d x .
$$

Therefore, the PDF function of the received signal in OCHM systems is given as

$$
\begin{aligned}
f_{Y}(y)= & P_{p} \cdot \frac{1}{\sqrt{2 \pi \sigma^{2}}} e^{-y^{2} / 2 \sigma^{2}} \\
& +\left(1-P_{p}\right) \cdot \frac{1}{\sqrt{2 \pi \sigma^{2}}} e^{-\left(y-\sqrt{E_{s}}\right)^{2} / 2 \sigma^{2}},
\end{aligned}
$$

where the received signal follows the POM. As noted before, POM provides the upper bound of the BER performance of OCHM systems. Furthermore, POM maintains the consistency according to the distance between the MU and the BS since the perforation effect is not dependent on the relative distance. The BER performance in POM provides the overall system performance regardless of the MU's location for a given perforation probability. Therefore, we utilize the POM for the analysis of BER performance in the rest of this paper.

\section{Performance Analysis}

\section{A. BER Performance of OCHM system with convolutional codes}

We consider the coded bit error probability of the OCHM systems with the convolutional codes (CC). CC is one of the most commonly used channel coding schemes in wireless communication systems. We assume that BPSK modulation 
is used for transmitting symbols over an AWGN channel with one-sided power spectral density $N_{0}$. For a soft-decision Viterbi decoder, the probability of error in the pairwise comparison of two paths at a node that differ in $d$ symbols is expressed as:

$$
P_{d}=P\left\{\sum_{r=1}^{d} y_{r} \leq 0\right\}
$$

where $y_{r}$ represents the $r$-th received symbol. Eq. (7) is called the first error event probability. If we assume an all-zero codeword is transmitted according to the following: $0 \rightarrow$ $+\sqrt{E_{s}}, Z=\sum_{r=1}^{d} y_{r}$ is the sum of $d$ independent Gaussian random variables, each with mean $\sqrt{E_{s}}$ and variance $N_{0} / 2$, that is, $Z$ is a Gaussian random variable with mean $d \sqrt{E_{s}}$ and variance $d N_{0} / 2$. Thus, Eq. (7) can be written as:

$$
P_{d}=Q\left(\sqrt{\frac{2 d E_{s}}{N_{0}}}\right)=Q\left(\sqrt{\frac{2 d R_{c} E_{b}}{N_{0}}}\right),
$$

where $R_{c}$ denotes the code rate of the encoder. From Eqs. (7), (8) the event- and bit-error probability bounds of convolutional codes in AWGN channels are expressed as [9]:

$$
\begin{aligned}
& P_{e}<\sum_{d=d_{\text {free }}}^{\infty} A_{d} Q\left(\sqrt{\frac{2 d R_{c} E_{b}}{N_{0}}}\right), \\
& P_{b}<\sum_{d=d_{\text {free }}}^{\infty} B_{d} Q\left(\sqrt{\frac{2 d R_{c} E_{b}}{N_{0}}}\right),
\end{aligned}
$$

where $A_{d}$ and $B_{d}$ are the number of codewords of weight $d$ and the total number of non-zero information bits on all weight- $d$ codewords, respectively. $d_{\text {free }}$ is the free distance of convolutional codes.

We use the characteristic function of a random variable for analysis. The characteristic function of Gaussian random variable $N\left(\mu, \sigma^{2}\right)$ is given as

$$
\Phi_{X}(w)=\exp \left\{j \mu w-\frac{1}{2} \sigma^{2} w^{2}\right\} .
$$

When we consider the POM case in OCHM systems, the characteristic function of the received signal in OCHM systems is obtained by

$$
\begin{aligned}
\Phi_{Y}(w)= & P_{p} \cdot \exp \left\{-\frac{1}{2} \sigma^{2} w^{2}\right\} \\
& +\left(1-P_{p}\right) \cdot \exp \left\{j \sqrt{E_{s}} w-\frac{1}{2} \sigma^{2} w^{2}\right\} .
\end{aligned}
$$

Then, the characteristic function of the random variable $Z$ is expressed as

$$
\Phi_{Z}(w)=\Phi_{Y_{1}}(w) \Phi_{Y_{2}}(w) \cdots \Phi_{Y_{n}}(w)=\left[\Phi_{Y}(w)\right]^{n},
$$

where each received signal $y_{i}$ in OCHM systems has the same probability density function and is independent of each other. Substitution of Eq. (12) into Eq. (13) yields the characteristic function of $Z$ as follows:

$$
\Phi_{Z}(w)=\sum_{i=0}^{n}\left(\begin{array}{c}
n \\
i
\end{array}\right)\left(1-P_{p}\right)^{i} P_{p}^{n-i} e^{j w \sqrt{E_{s}} \cdot i-\frac{\sigma^{2} w^{2} n}{2}}
$$

Therefore, the probability density and distribution functions of $Z$ are obtained as

$$
f_{Z}(z)=\sum_{i=0}^{n}\left(\begin{array}{c}
n \\
i
\end{array}\right)\left(1-P_{p}\right)^{i} P_{p}^{n-i} \frac{1}{2 \pi n \sigma^{2}} e^{\frac{-\left(z-i \sqrt{E_{s}}\right)^{2}}{2 n \sigma^{2}}}
$$

and

$$
F_{Z}(z)=\sum_{i=0}^{n}\left(\begin{array}{c}
n \\
i
\end{array}\right)\left(1-P_{p}\right)^{i} P_{p}^{n-i} \cdot G\left(\frac{z-i \sqrt{E_{s}}}{\sqrt{n} \sigma}\right)
$$

From Eqs. (12) through (16), the first event error probability of the convolutional codes in OCHM systems is expressed as:

$$
P_{d, C C}^{O C H M}=\sum_{i=0}^{d}\left(\begin{array}{c}
d \\
i
\end{array}\right)\left(1-P_{p}\right)^{i} P_{p}^{d-i} \cdot Q\left(\sqrt{\frac{2 i^{2} R_{c} E_{b}}{d N_{0}\left(1-P_{p}\right)}}\right) .
$$

Hence, the event- and bit-error probability bounds in OCHM systems with the convolutional codes are expressed as:

$$
\begin{aligned}
P_{e, C C}^{O C H M}< & \sum_{d=d_{\text {free }}}^{\infty} A_{d} \sum_{i=0}^{d}\left(\begin{array}{c}
d \\
i
\end{array}\right)\left(1-P_{p}\right)^{i} P_{p}^{d-i} \\
& \cdot Q\left(\sqrt{\frac{2 i^{2} R_{c} E_{b}}{d N_{0}\left(1-P_{p}\right)}}\right)
\end{aligned}
$$

and

$$
\begin{aligned}
P_{b, C C}^{O C H M}< & \sum_{d=d_{\text {free }}}^{\infty} B_{d} \sum_{i=0}^{d}\left(\begin{array}{c}
d \\
i
\end{array}\right)\left(1-P_{p}\right)^{i} P_{p}^{d-i} \\
& \cdot Q\left(\sqrt{\frac{2 i^{2} R_{c} E_{b}}{d N_{0}\left(1-P_{p}\right)}}\right)
\end{aligned}
$$

where $A_{d}$ and $B_{d}$ are determined by the encoder structure.

\section{B. Frame error rate (FER) analysis from the BER perfor- mance analysis}

In data communications, FER is important because even a single bit error in a frame may make the frame useless. Thus, most mobile communication systems choose the FER as a QoS parameter. A reasonable approximation for FER of a channel coding scheme is given by

$$
\begin{aligned}
F E R & \simeq 1-\left(1-P_{b}\right)^{L_{\text {frame }}} \\
& \simeq P_{b} \cdot L_{\text {frame }},
\end{aligned}
$$

where $P_{b}\left(P_{b} \ll 1\right)$ and $L_{\text {frame }}$ denote the bit error probability of a channel coding scheme and the frame length, respectively.

\section{Downlink power allocation for OCHM systems}

In OCHM systems, the downlink power which is allocated for a specific MU is determined by the perforation probability, $P_{p}$, as well as the channel environment. The perforation probability varies according to the number of users in a cell and the user activity at the specific time. Previous work only considered the mean perforation probability according to the number of MUs in a cell. However, the perforation probability of each MU is different from each other. Some MUs have 
lower or higher perforation probabilities than others at the specific time. In downlink, BS can know the exact number of perforated symbols in a frame of each MU at the time. Through the BER performance analysis shown in Section IIIA, BS can determine the allocated power for a specific MU in downlink. If the BER or FER performance analysis does not exist, the link-level simulation results are required in each case.

The additional required $E_{b} / N_{0}$ at an $\mathrm{MU}$ due to the perforation can be expressed as:

$$
\Delta\left(E_{b} / N_{0}\right)=f\left(F E R_{r e q}, P_{p}\right)-f\left(F E R_{r e q}, 0\right),
$$

where $f(A, B)$ denotes the function of the required $E_{b} / N_{0}$ at MU for $F E R_{r e q}=A$ and $P_{p}=B$, and it is derived from Eqs. (19) and (20). The last term in Eq. (21) indicates the required $E_{b} / N_{0}$ for $P_{p}=0 . F E R_{r e q}$ denotes the required FER at receiver. The allocated symbol power for a specific $\mathrm{MU}$ at $\mathrm{BS}$ is determined as:

$$
P_{T}=\frac{f\left(F E R_{r e q}, P_{p}\right) \cdot \mu R_{c} \cdot I_{\text {total }}}{T_{s} \cdot L_{\text {path }}},
$$

where $\mu, I_{\text {total }}, T_{s}$, and $L_{\text {path }}$ denote the encoded bits per modulated symbol, the total interference at MU, the symbol time, and the path-loss between BS and the MU, respectively. As $P_{p}$ increases, the required energy at receiver, $f\left(F E R_{r e q}, P_{p}\right)$, increases and the allocated power, $P_{T}$, also increases.

\section{User capacity analysis}

OCHM was proposed to accomodate more MUs than the number of codewords in downlink of CDMA systems. Recently, we have analyzed the downlink user capacity of OCHM system and have compared it with the user capacity of the conventional CDMA system [6]. In [6], we defined code and power capacities independently like as the conventional CDMA systems. Thus, the overall user capacity is derived by the minimum value of two capacities. Code capacity of OCHM systems is defined as the maximum number of HPs for a given $P_{p}$, which is expressed as:

$$
M_{c}=1+\frac{\ln \left(1-P_{p}\right)}{\ln \left(1-\frac{(s-1) \cdot \bar{v}}{s \cdot N_{O C}}\right)} .
$$

It is derived from Eq. (2). Power capacity is defined as the maximum number of users under the condition that the allocated power is less than or equal to the maximum available power at BS, and it is expressed as:

$$
M_{p} \leq \frac{E_{s, \text { max }} \cdot \bar{L}_{\text {path }}}{\bar{v} \cdot\left(\frac{E_{b}}{I_{0}}\right)_{\text {target }}^{\text {OCHM }} \cdot \mu R_{c} \cdot I_{\text {total }}},
$$

where $E_{s, \max }, \bar{L}_{\text {path }}$, and $\left(E_{b} / I_{0}\right)_{\text {target }}^{O C H M}$ denote the maximum symbol energy for data traffic at BS, the average pathlass at an $\mathrm{MU}$, and the target $\left(E_{b} / I_{0}\right)$ for a given FER at MU, respectively. In [6], we have illustrated the user capacity which is the minimum value of Eqs. (23) and (24) for varying $\left(E_{b} / I_{0}\right)_{\text {target }}^{O C H M}$ and $P_{p} .\left(E_{b} / I_{0}\right)_{\text {target }}^{O C H M}$ was evaluated through

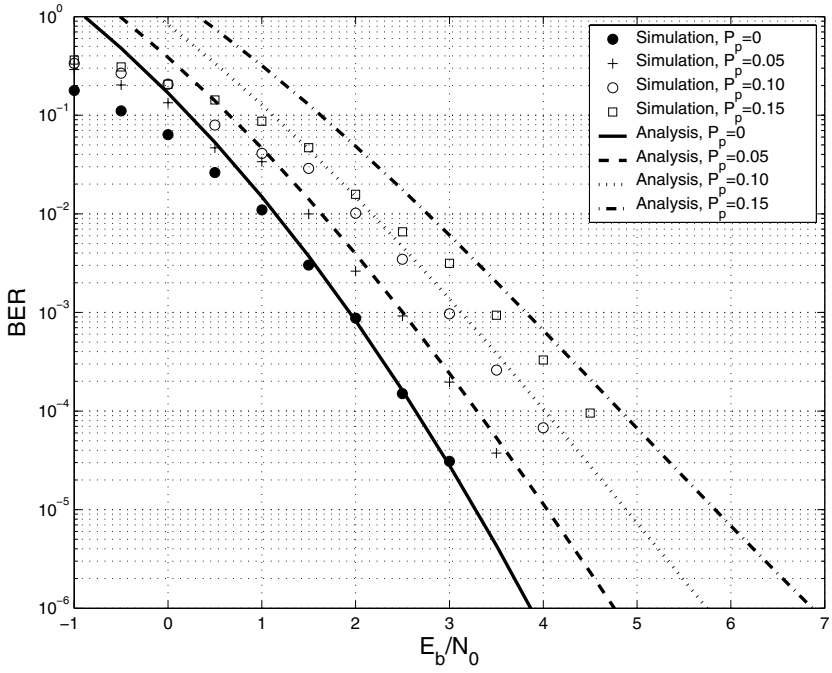

Fig. 2. BER performance of CC, $K=9, R_{c}=\frac{1}{3}$

computer simulations for several cases in [6]. However, as we noted in Section III-C, the additional required energy at MU increases as $P_{p}$ increases. The allocated power for a specific MU in downlink at BS also increases as $P_{p}$ increases. Thus, the $\left(E_{b} / I_{0}\right)_{\text {target }}^{\text {OCHM }}$ value in Eq. (24) is dependent on $P_{p}$. In fact, $\left(E_{b} / I_{0}\right)_{\text {target }}^{O C H M}=f\left(F E R_{r e q}, P_{p}\right)$ and the power capacity of OCHM system is expressed as:

$$
\tilde{M}_{p} \leq \frac{E_{s, \max } \cdot \bar{L}_{\text {path }}}{\bar{v} \cdot f\left(F E R_{\text {req }}, P_{p}\right) \cdot \mu R_{c} \cdot I_{\text {total }}},
$$

where $f\left(F E R_{r e q}, P_{p}\right)$ is determined by the channel coding capability.

\section{NUMERICAL EXAMPLES}

\section{A. BER performance}

We assume that the wireless channel is an AWGN channel and a BPSK modulation scheme is utilized. In CDMA systems, a wireless channel can be AWGN even when multi-paths exist since the de-correlated output of interference at receiver can be modeled as a gaussian random variable. In addition, the analysis for BPSK can be used for QPSK. Fig. 2 shows the BER performance of convolutional code in OCHM systems. We utilize a convolutional code with a code rate of $1 / 3$ and a constraint length of $9(K=9)$ using a generator polynomial $(557,663,711)$ in octal number, and then, the free distance $\left(d_{\text {free }}\right)$ of the encoder is 18 . The convolutional code introduced above was used in the IS-95 uplink system [10]. The solid line represents Eq. (10) in case that $P_{p}=0$, and the broken lines represent Eq. (19) in case that $P_{p} \neq 0$. The symbols with marks indicate the results of computer simulations. The results of Eqs. (10) and (19) show the upper bound of the simulation results. The BER values increase as the perforation probability increases. When the $P_{p}$ value is set to 0.10 , the additional required $E_{b} / N_{0}$ is approximately $1.5 \mathrm{~dB}$ for $10^{-4}$ BER performance. 


\section{B. Additional required energy at $M U$}

Table I summarizes the required $E_{b} / I_{0}$ of convolutional code in Section IV-A for varying the values of $F E R_{r e q}$ and $P_{p}$. As noted before, the required $E_{b} / N_{0}$ values increase as $P_{p}$ increases and BS should allocate more power to MUs with large values of $P_{p}$. More allocated power to MUs can limit the power capacity. Therefore, the channel coding scheme used for OCHM is a key factor affecting OCHM performances. If an MU requires larger power than a limit due to its perforation, BS need to perform an appropriate admission control scheme in order to save power consumption.

TABLE I

REQUiRED $E_{b} / I_{0}$ AT MU ACCORDING TO $P_{p}$ AND $F E R_{r e q}$

\begin{tabular}{|c|c|c|c|c|}
\hline \multicolumn{5}{|c|}{ CC with $\mathrm{K}=9$ and $L_{\text {frame }}=1024$} \\
\hline$F E R_{\text {req }}$ & $P_{p}=0.00$ & $P_{p}=0.05$ & $P_{p}=0.10$ & $P_{p}=0.15$ \\
\hline $10 \%$ & $2.63 \mathrm{~dB}$ & $3.28 \mathrm{~dB}$ & $4.02 \mathrm{~dB}$ & $4.82 \mathrm{~dB}$ \\
$1 \%$ & $3.30 \mathrm{~dB}$ & $4.03 \mathrm{~dB}$ & $4.89 \mathrm{~dB}$ & $5.81 \mathrm{~dB}$ \\
\hline
\end{tabular}

\section{User capacity}

OCHM systems mitigate the code limit situation by allowing HP collision between active MUs. However, the HP collisions degrade the link level performance. Thus, each MU requires more energy for the same FER performance, compared with that of the conventional systems, as shown in Section IV-B. It decreases the power capacity. Since the overall capacity is the minimum value of code capacity and power capacity, the additional required energy at $\mathrm{MU}$ according to $P_{p}$ is the most important factor which determines the capacity. The code capacity of OCHM systems shown in Eq. (23) increases as $P_{p}$ increases, but the power capacity of OCHM system as shown in Eq. (25) decreases as $P_{p}$ increases. Hence, the overall capacity is determined by the intersection point of two user capacity curves. Fig. 3 shows the user capacities for varying the OCI values with $\mathrm{CC}$ as shown in IV-A. As the OCI value increases, the user capacity decreases since $\tilde{M}_{p}$ decreases. Table II summarizes the parameters and the overall user capacities of OCHM systems for varying the OCI values. The other parameters for modelling interference have the same values as those used in [6] and we assume that an omni-antenna is used at BS. When the OCI value is set to $20 \%$, 281 users can be accommodated in downlink, which is much larger than the number of orthogonal codewords $\left(N_{o c}=64\right)$.

\section{TABLE II}

OVERALL USER CAPACITIES OF OCHM SYSTEMS FOR VARYING OCI

\begin{tabular}{|c|c|c|c|c|c|}
\hline \multicolumn{5}{|c|}{$F E R_{r e q}=10 \%, L_{\text {frame }}=1024, \bar{\nu}=0.1, N_{O C}=64, \mu=2$} \\
\hline OCI & $20 \%$ & $40 \%$ & $60 \%$ & $80 \%$ & $100 \%$ \\
\hline capacity & 281 & 186 & 133 & 114 & 90 \\
\hline
\end{tabular}

\section{CONCLUSIONS}

In this paper, we introduce POM as a received signal model for OCHM systems. POM provides an upper bound of the BER in OCHM systems. The BER performance of OCHM systems is analyzed. The analytical results of coded OCHM system show the upper bound of the computer simulation results. Through the analytical results, we can compute the additional

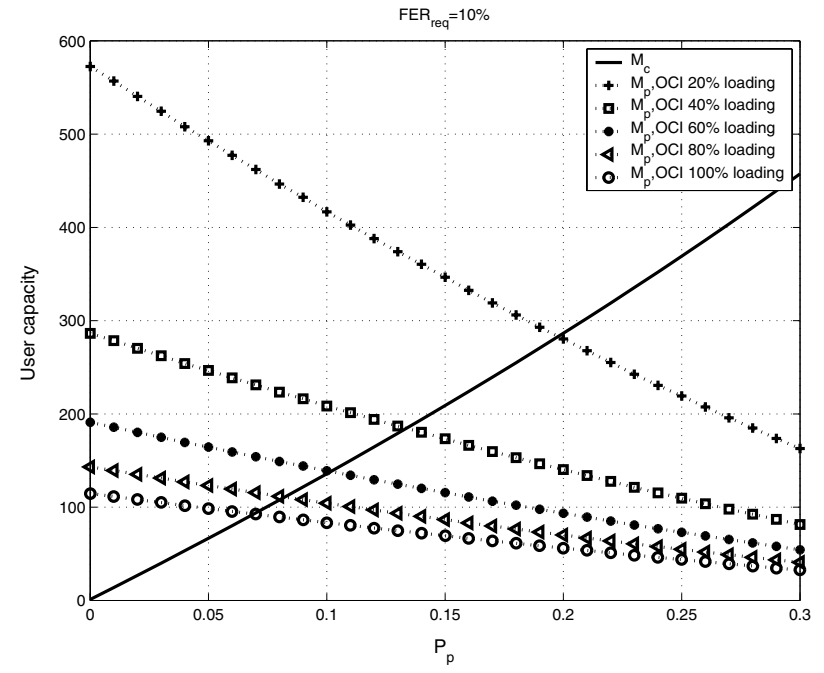

Fig. 3. User capacities of OCHM system for varying the OCI values of CC with $\mathrm{K}=9$

required energy at the receiver due to the perforation effect and adjust the power level for a target BER/FER performance at the receiver for varying the perforation probability. In addition, the user capacity of OCHM can be estimated for a given channel coding scheme.

\section{ACKNOWLEDGMENTS}

This research was supported in part by BroMA IT Research Center Project.

\section{REFERENCES}

[1] 'Physical layer aspects of UTRA High Speed Downlink Packet Access (Release 4)', 3GPP TR25.848 V4.0.0, Mar. 2001.

[2] 'cdma2000 High Rate Packet Data Air Interface Specification', 3GPP2. C.S0024 v.4.0, Oct., 2000

[3] S. Park and D. K. Sung, "Orthogonal code hopping multiplexing," IEEE Communi. Lett., Vol. 6, No. 12, pp.529-531, Dec. 2002.

[4] J. K. Kwon, S. Park, and D. K. Sung, "Log-likelihood conversion schemes in orthogonal code hopping multiplexing," IEEE Commun. Lett., Vol. 7, No. 3, pp.104-106, Mar. 2003.

[5] J. H. Chung, S. Park, and D. K. Sung, "Symbol perforation reduction schemes for orthogonal code hopping multiplexing," IEICE Trans. on Commun., (Accepted).

[6] S. H. Moon, S. Park, J. K. Kwon, and D. K. Sung, "Capacity improvement in CDMA downlink with orthogonal code hopping multiplexing," IEEE Trans. on Vehicular Technology,(Accepted).

[7] J. K. Kwon, S. Park, and D. K. Sung, "Collision mitigation by loglikelihood ratio (LLR) conversion in orthogonal code hopping multiplexing," IEEE Trans. on Vehicular Technology,(Accepted).

[8] E. A. Geraniotis and M. B. Pursley, "Error probability for slow-frequencyhopped spread-spectrum multipel-access communications over fading channels," IEEE Trans. Commun., Vol. 30, No. 5, pp.996-1009, May 1982.

[9] A. J. Viterbi, "Convolutional codes and their performance in communication systems," IEEE Trans. Commun., Vol-COM. 19, No. 5, pp. 751-772, Oct. 1971.

[10] J. S. Lee and L. E. Miller, CDMA systems engineering handbook, Artech House Publishers, 1998 